\title{
A Local Application of Climate Niche Models for Chinese Fir and Possible Adaptation Strategies under a Changing Climate -- A Case Study in Fujian Province, China
}

\author{
Haijun Kang, Biyun Xiao, Ling Chen \\ Fuzhou University of International Studies and Trade, Fuzhou, Fujian Province, China
}

Keywords: Chinese fir (Cunninghamia lanceolata); climate niche; Random Forest; climate change; adaptation strategies

\begin{abstract}
Climate niche models have been widely used to assess the potential impacts of climate change on the climatic suitability of tree species and ecosystems. This paper presents a case study of local applications of this approach at a study area in Fujian province for Chinese fir. ClimateAP is applied to assess the impact of climate change on this species at a fine scale $(25 \times 25 \mathrm{~m})$. Inventory data are used to examine the applicability of the model prediction at the study area. Seven selected climate change scenarios are used to generate individual and consensus projections of the climate niche of this species for the 2020s, 2050s and 2080s. The result suggests that climate change will lead to a dramatic contraction of the spatial extent of the climate niche for Chinese fir. A spectrum of adaptation strategies is proposed based on these projections considering the nature of the projections being the realized climate niche.
\end{abstract}

\section{Introduction}

The impact of climate change on forest ecosystems has become increasingly evident over the past decade[1,2] and is now widely recognized as one of the most important threats to the capacity of forest landscapes to provide ecological, economic and social services. Climate is one of the major factors affecting the geographic distribution of forest ecosystems and tree species [3].

Chinese fir (Cunninghamia lanceolata (Lamb.) Hook), a characteristic subtropical coniferous species in China, is one of the most important indigenous and commercial tree species for timber production [4]. In the context of global climate change, a number of studies have indicated that the productivity and geographical distribution of Chinese fir may change significantly in the future, with significant implications for its productivity [5-7]. However, predictions of these studies are at species level and at spatial resolutions that are not detailed enough for developing adaptive local forest management strategies. In addition, the accuracy of their predictions at local scale is unknown and need to be validated.

Fujian Province is one of the most important Chinese fir production regions. Shunchang County, located in the north of Fujian, is at the center of the distribution of Chinese fir. A study of the climate niche shifts for Chinese fir in response to climate change and an analysis of possible adaptive management strategies at an operational scale in this area is particularly important.

\section{Methods}

\subsection{Study Area.}

The study area is located in Shunchang County in the north of Fujian Province $\left(117^{\circ} 29^{\prime}-118^{\circ} 14^{\prime}\right.$ $\mathrm{E}$ and $26^{\circ} 38^{\prime}-27^{\circ} 12^{\prime} \mathrm{N}$ ). The area of Shunchang is $1,985 \mathrm{~km} 2$. It has a subtropical maritime monsoon climate, but is also influenced by the continental climate to the west. The annual average temperature in the study area is $16.9^{\circ} \mathrm{C}$, the frost-free period lasts 305 days and the average annual rainfall is $1628 \mathrm{~mm}$. With a mild and humid climate and ample sunshine and rainfall, Shunchang provides ideal conditions for Chinese fir. Shunchang County Forestry Bureau issued the permission 
to conduct the study on this site. The specific study area includes Shuangxi Town and Yangkou Town, which have a total area of 29,360 hectares.

\subsection{The Climate Niche Model.}

The climate niche model used in this study was recently developed in a previous study [7]. The model was constructed based on presence and absence data of the species from a digital version of Vegetation Map of China (1:1,000,000). Spatially explicit climate data were generated for the study area using ClimateAP. The RF algorithm was used to build the model.

\subsection{Climate Data.}

We used ClimateAP, an extension of ClimateWNA[8], to generate the climate data for this study. The future climates were generated using seven climate change scenarios to project future distributions of Chinese fir, including three IPCC AR5 GCMs (CNRM-CM5, HadGEM2 and CCSM4) in combination with three emission scenarios (RCP2.6, RCP4.5 and RCP8.5) to represent an "aggressive mitigation", a "low emission" and a "business as usual" scenario, respectively. There was only one GCM with RCP2.6 available in ClimateAP model as it is focused on RCP4.5 and RCP8.5. The generated datasets were processed to meet the format required for model input to project future climate niches for Chinese fir in the study area.

\subsection{Application of the Climate Niche Model.}

The RF climate niche models were fed with climate variables for the gridded data points to predict the spatial distribution of the climate niche for Chinese fir for the reference period and the three future periods for the seven climate change scenarios. For the reference period, the final predictions were the consensus over the outputs of the 10 RF models. For each data point, if five or more of the RF models predicted presence, the species was considered to be present. For the future periods, the same approach was applied to each individual climate change scenario. The final consensus predictions were obtained by taking the consensus across the seven climate change scenarios. The predictions were then imported into ArcGIS (V10.2.1) to generate maps.

\subsection{Forest Inventory Data.}

Forest inventory data were obtained from a spatial forest resources survey undertaken in 2007 in Shunchang County. These inventory data include coverages for all plant species, non-forest land and water bodies. The dominant species include Chinese fir (17, 199 ha, 59\% of the study area), bamboo (4,401 ha, 15\%) and some economic forests (982 ha, 3\%). Water bodies accounts for 19\% of the total area, which were excluded from the comparisons. The study area is favorable for both Chinese fir and Bamboo. Due to the increase in economic values of the bamboo forest, however, the area of bamboo plantation expanded substantially in past several decades.

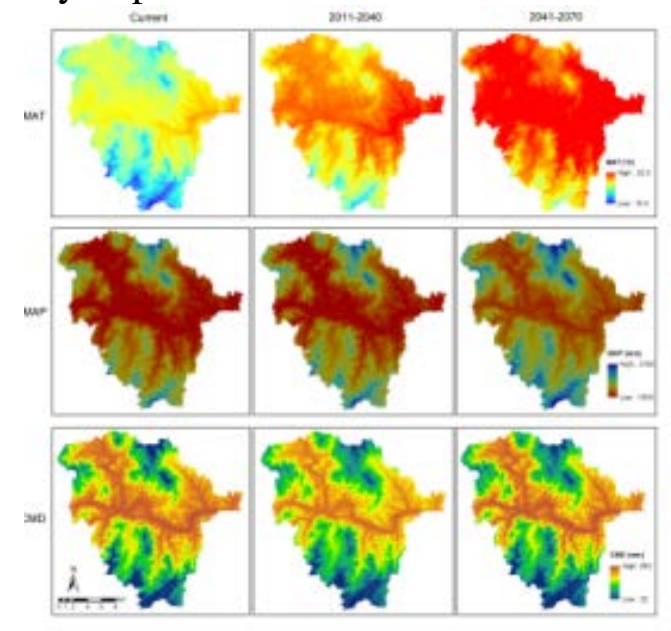

Fig. 1. Distribution of mean annual temperature (MAT), mean annual precipitation (MAP) and cumulative moisture deficit (CMD). 


\section{Results}

\subsection{Projections of Future Climate Regimes.}

Temperature and precipitation are the main climatic factors that impact the geographic distribution of Chinese fir. The distributions of mean annual temperature (MAT), mean annual precipitation (MAP) and cumulative moisture deficit (CMD) for the current period, 2020s and 2050s projected by one of the 7 selected climate change scenarios (HadGEM2 RCP4.5) are shown in Fig. 1. Predictions of climate change using other climate change scenarios are not presented here. The changes in MAT in the future are more obvious than the changes in MAP and the changes in CMD are somewhat in between. The effect of temperature is more important than precipitation for Chinese fir in Fujian Province. Thus, increasing temperature in the future may considerably impact the suitability of the climatic conditions for this species.

\subsection{Predicted Climate Niche vs. Current Species Distribution.}

The climate niche predicted for the reference period and current distribution of Chinese fir are shown in Fig. 2. The predicted climate niche encompassed the entire study area while the actual distribution of the species occupied $72 \%$ of the non-water land-base in the study area. The remaining area (28\%) was mostly occupied by bamboo (19\%) and other economic forests (4\%). The high level of agreement between the predicted and observed presence of Chinese fir suggest that the climate niche model developed for this species is applicable to the study area.

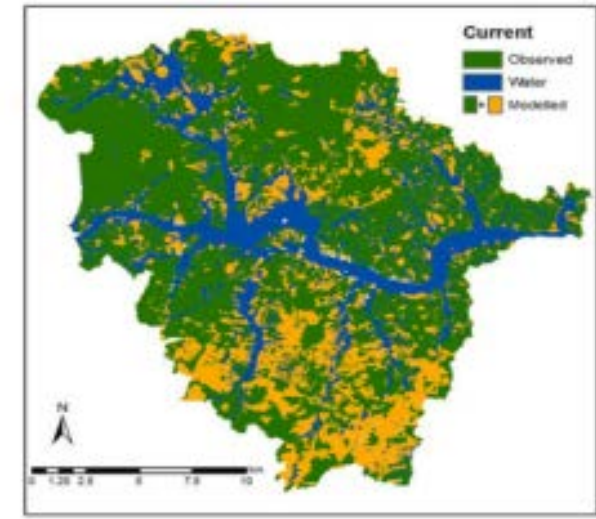

Fig. 2. Consensus prediction of the climate niche for Chinese fir for the current period.

\subsection{Consensus Projections of the Climate Niche for Chinese Fir in Future Periods.}

Projections of the climate niche for Chinese fir for the 2020s, 2050s and 2080s under different climate change scenarios are shown in Figs. 3, 4 and 5. The projections for each future period vary among the different climate change scenarios used. In the 2020s, the projected results are consistent, with no major range contraction except for some aggressive climate change scenarios (See Fig. 3B, 3C and 3D). The consistency declined sharply in the 2050s and 2080s. In the 2050s, most of the climate change scenarios suggest that a substantial proportion of the study area may be unsuitable for Chinese fir. Two scenarios (Fig. 4E, 4F) were exceptions; these two scenarios show a low annual globally averaged surface air temperature anomaly. The results for the 2080s are more severe, with only scenario CCSM4_rcp26 (Fig. 5E) suggesting that some of the study area will be suitable for Chinese fir. This is because in the CCSM4 RCP2.6 scenario, there is a stabilizing trend in the 2050s-2080s in line with the target of keeping warming below the $2^{\circ} \mathrm{C}$ threshold above preindustrial temperatures. 


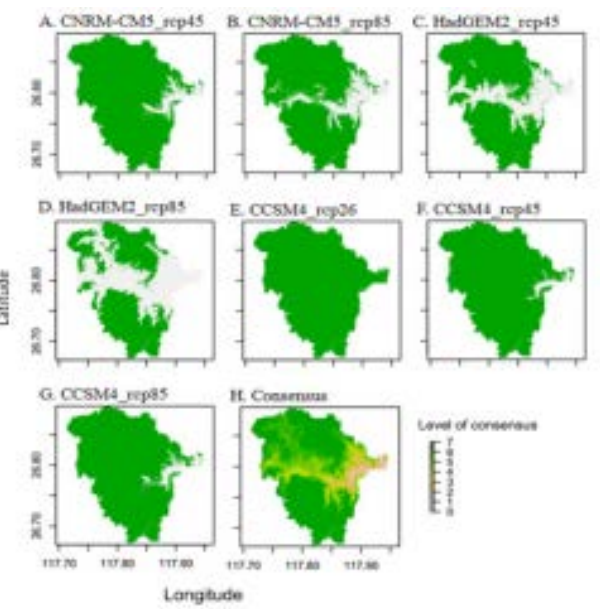

Fig. 3. The projections for the climate niche of Chinese fir in the 2020s using different climate change scenarios. The number (0-7) in the legend (the color scheme) indicates the level of consensus among the seven selected climate change scenarios.

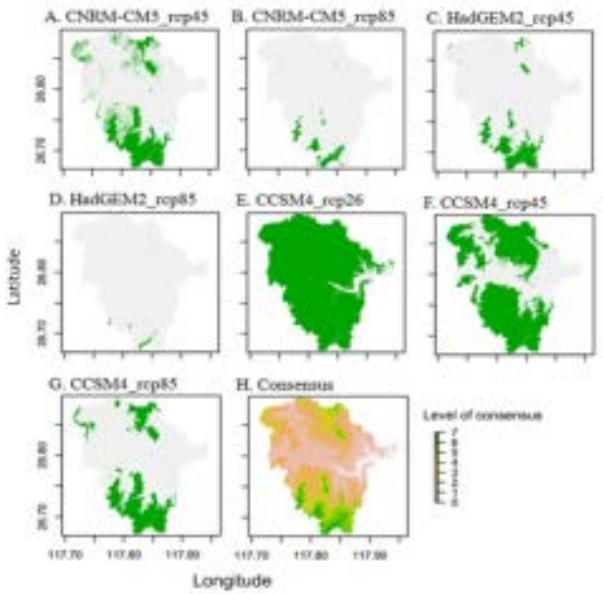

Fig.4. The projections for the climate niche of Chinese fir in the 2050s using different climate change scenarios.
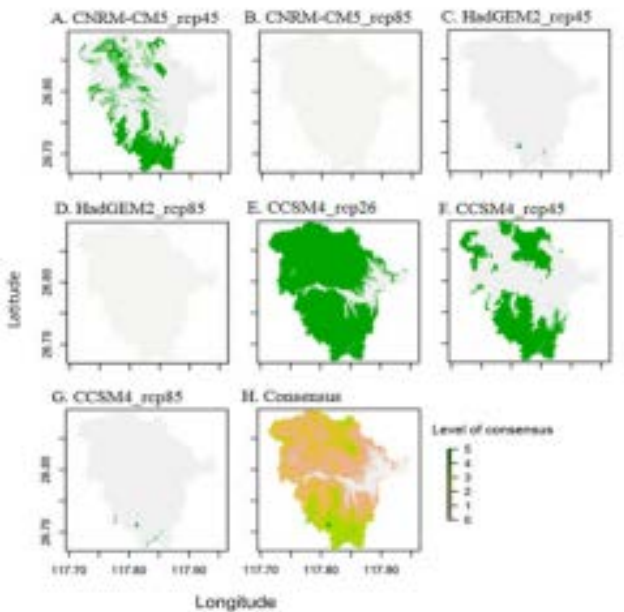

Lenghude

Fig.5. The projections for the climate niche of Chinese fir in the 2080s using different climate change scenarios. 


\section{Discussion}

\subsection{Application of the Climate Niche Models.}

The climate niche models developed for this species predict that the entire study area is within the climate niche of Chinese fir. However, about $72 \%$ is actually occupied by this species based on the forest inventory data. For the remaining $28 \%$ of the areas, $19 \%$ of bamboo plantations and $4 \%$ of the economic forests are results of human intervention and likely represent false absences. Thus, the model prediction for the climate niche of this species is highly applicable for this study area at a high spatial resolution ( $25 \times 25 \mathrm{~m})$. This result provides additional support for the original species-level climate niche model built using Random Forest. Furthermore, it provides a solid foundation for developing adaptive management strategies at a local operational scale.

\subsection{Consensus Projections for Future Periods.}

The results indicate that the geographic distribution of the climate niche for Chinese fir in the study area is projected to substantially contract and may even disappear in the future. The results of our study show that changes in the climate niche of Chinese fir vary with different greenhouse gas emission scenarios. In the "business as usual" scenario (RCP8.5), the distribution of Chinese fir within the study area will contract markedly in the 2050s and may even disappear in the 2080s. However, in the "aggressive mitigation" scenario (RCP2.6), the contraction is limited to $5.4 \%$ in the 2050 s and $12.4 \%$ in the 2080s. Such results indicate that controlling CO2 concentrations in the atmosphere will have important implications for the distribution of Chinese fir.

\subsection{Adaptation Strategies for Chinese Fir Management under a Changing Climate.}

Species Selection. A direct application of climate niche model projections is species selection or assisted migration. Assisted migration is often necessary for the areas with projected range expansion or contraction. For the area in this study, range expansion was not projected to occur as the entire study area is currently within the climate niche of this species. However, range contraction was projected to be the main outcome. Therefore, selection of species for plantation in the future needs to consider other forest tree species. Masson pine (Pinus massoniana) could be a candidate. Masson pine occupies similar climate conditions, but has a slightly broader climate niche than Chinese fir and is projected to persist in this region.

Provenance Selection. A considerable amount of among-population genetic variation exists within a species and different populations may respond differently to climate change [9]. Provenance tests were conducted for Chinese fir at regional and national scales [10]. It is expected that some populations will be more suitable for the study area under future climates. Previous studies have indicated that improved varieties are tolerant of a wider range of climatic conditions and the use of hybridization and genetic engineering could increase the genetic diversity of improved varieties, which could increase the survival ability of tree species under a changing climate [11]. Forest managers could also select unrelated or distant families from near-natural forests or unmodified parent stands for cross-breeding tests.

Developing Mixed Forests. Forest managers have traditionally preferred pure stands of Chinese fir as these result in better economic yields. However, the genetic diversity index in pure Chinese fir plantations is low. The crown types and community physiognomy of pure Chinese fir plantations make them less capable to adjust to changes in climatic factors, such as temperature, humidity, radiation, and wind[11]. As a result, the adaptiveness of pure Chinese fir plantations to climate change or extreme weather conditions is poor. Compared to pure plantations, mixed forests have greater resilience and higher species diversity, and as a result they are better able to adapt to climate-induced stressors and disturbances, such as forest fire, disease or insect outbreaks [11]. It would be appropriate to mix some tree species with a broader climate niche with Chinese fir to create mixed forests.

Forest Monitoring. As the fundamental niche of this species is unknown, the performance of the 
trees growing outside their realized niche is worth monitoring. With the continuous improvement of sustainable forest management implementation and computer technology, the development of resource information management and service network systems for the dynamic monitoring of forest resources has become an important part of the adaptive management of forests. Forest managers need to strengthen the monitoring of forest resources so that they can be informed when serious impacts on growth and survival occur in the future.

\section{Conclusion}

This study focused on a local application of climate niche models for Chinese fir at a study area in Fujian province. We examined the applicability of the species range-wide model at the study area and projected changes in the climate niche at a fine spatial resolution for future periods. Results suggest that the geographic distribution of the suitable climate niche for Chinese fir in the study area will contract dramatically and the species may even disappear in the future. This finding is alarming as the study area is located in a central productive area of the species. A spectrum of adaptive strategies were proposed and discussed, including assisted migration at species and population levels under different circumstances. As the fundamental niche of this species is unknown, performance of the trees growing outside their realized niche will be interesting to monitor under a changing climate.

\section{References}

[1] T. Wang, E.M. Campbell, G.A. O'Neil, S.N. Aitken, Projecting future distributions of ecosystem climate niches: Uncertainties and management applications. Forest Ecol. Manag. 279 (2012) 128-140.

[2] W.A. Kurz, C.C. Dymond, G. Stinson, G.J. Rampley, E.T. Neilson, A.L. Carroll, T. Ebata, L. Safranyik, Mountain pine beetle and forest carbon feedback to climate change. Nature. 452 (2008) 987-990.

[3] D.W. McKenney, J.H. Pedlar, K. Lawrence, K. Campbell, M.F. Hutchinson, Potential Impacts of Climate Change on the Distribution of North American Trees. BioScience. 57 (2007) 939-948.

[4] B. Wang, W. Wei, Z. Xing, W. You, X. Niu, X. Ren, C. Liu, Biomass carbon pools of Cunninghamia lanceolata (Lamb.) Hook. forests in subtropical China: Characteristics and potential. Scand. J. Forest Res. 27 (2012) 545-560.

[5] J. Liu, F. Kang, Potential impact of climate change on distribution of Cunninghamia lanceolata. J. Southwest Forest Univ. 30 (2010) 22-24,32. (in Chinese)

[6] W. Shi, H. Tong, Prediction of potential distribution of Chinese fir in Guizhou next 70 years and ecological countermeasures. J. Central South Univ. Forest Technol. 33 (2013) 87-92. (in Chinese)

[7] T. Wang, G. Wang, J. Innes, C. Nitschke, H. Kang, Climate niche models and their consensus projections for future climates for four major forest tree species in the Asia-Pacicifc region. Forest Ecol. Manag. 360 (2016) 357-366.

[8] T. Wang, A. Hamann, D.L. Spittlehouse, T.Q. Murdock, ClimateWNA-High-Resolution Spatial Climate Data for Western North America. J. Appl. Meteorol. Climatol. 51 (2012) 16-29.

[9] T. Wang, A. Hamann, A. Yanchuk, G.A. O'neil, S.N. Aitken, Use of response functions in selectiong lodgepole pine populations for future climates. Global Change Biol. 12 (2006) 2404-2416.

[10] Y. You, J. Hong, Application of RAPD marker to genetic variation of Chinese fir provenances. Scientia Silvae Sinicae. 34 (1998) 32-38. (in Chinese)

[11] Q. Guo, S. Liu, L. Chen, Z. Shi, Adaptation strategy of forestry to climate change in China. Chin. J. Ecol. 15 (1996) 47-54. (in Chinese) 